\title{
Word Level Similarity Auto-Evaluation for an Online Question Answering System
}

\author{
DOI : 10.36909/jer.ICETET.14991 \\ Madhav A. Kankhar, C. Namrata Mahender* \\ Department of Computer Science \& Information Technology, Dr. Babasaheb Ambedkar \\ Marathwada University Aurangabad. \\ *Email: madhavkankhar.07@gmail.com; cnamrata.csit@bamu.ac.in.
}

\begin{abstract}
Over the last decade, the educational system has undergone numerous changes, the most significant of which is the shift in learning and examination methods. Educational institutions are gradually going into online instruction and education, which is altering students' and parents' perceptions of learning. The scenario of Covid-19 has seriously raised demand for online subjective examination and evaluation process to be automated. Paraphrasing is the challenge while designing and evaluation an fully automated Question Answering System. This paper has shown the word level similarity for evaluation one word answer of fill in the blank types of question taken in real time scenario.
\end{abstract}

Key words: Online Question Answering System (QAS); NLP; General Components of QA System;

\section{INTRODUCTION}

Question answering is a type of information retrieval technique that is unique (Dhokrat, Asmita, et al., 2012). The purpose of the QuestionAnsweringSystem is to find the correct answer to the questionpose in naturallanguage. Natural Language Processing focuses on computer-to-computer communication in the context of stochastic outcomes and experimental applications (Gupta Poonam, et al.,2012). 
The question answering system is divided by two categories open domain and close domain, open domain question answering system is dealing with unrestricted topics that are the question forward any subject the open domain question answering system tackles large size of data to extract the most relevant answer. And second one is close domain question answering system, it deal with the question is under the specific domain, that means the topic of question is restricted type, close domain question answering system are accepted only a limited type of question, this type of question answering is easy to handle, close domain question answering system example where it is used is medical and railways field.

Web-based question-answering systems, Information Retrieval(IR)/Information Extract(IE)based question-answering systems, Restricted domain question-answering systems, and Rulebased question-answering systems are all examples of question-answering systems. Many webbased Question Answering Systems (QAS) are being used these days, such as Google, Yahoo, and Ask. Wh-type queries, such as who, where, and how, are handled by web-based Question Answering systems. Example "When was A. P. J. Abdul Kalam born?". IR/IE utilized information retrieval technology to get back exact answer after supervision the document .Restricted domain question answering systems were built to progress the exactness of Question Answering systems. Rule based Question Answering systems will be giving rules for each type of questions like, who, why, where, where, when etc.

For example when is utilized to retrieve an answer which has time tag associated. Rule Based Question Answering system will progress the accuracy of the system (Vijoy, Maria et al., 2016). 


\section{Basic Architecture of QAS}

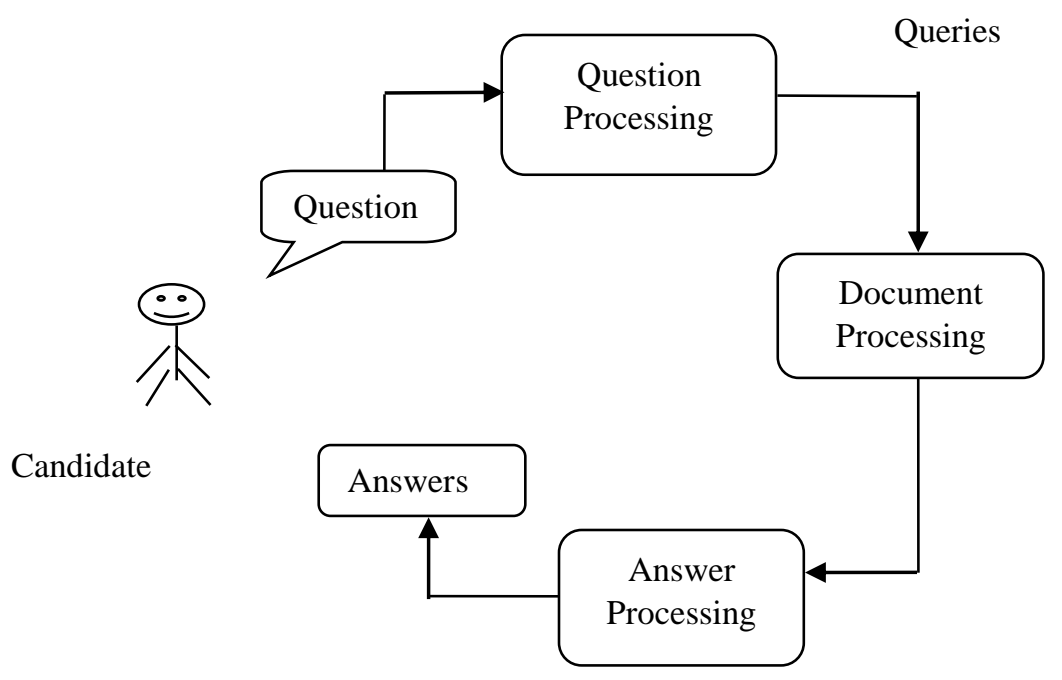

Fig. 1Basic Architecture of Question Answering System

The basic architecture of a question answering system discuss below.

\section{1) Question}

A question (https://en.wikipedia.org/wiki/question) is an expression that normally works as a request for information that is intended to be delivered in the form of an answer. As shown in Fig 1, the candidate asks a question to the system. Different types of questions are categorised, including those that begin with who, when, what, where, how, and why. Questions with the words what, when, who, and where are classified as factoid questions, while questions with the words why and how are classified as non-factoid questions. Generally, factoids are asked in the form of questions.

less demanding to prepare and are responded to/ answered in a single sentence (Allam, Ali Mohamed Nabil,et al., 2012).

\section{2) Question Processing}


The user asks any question for the system which is the main goal to identify the focus of question, it has too also classify the question type, expected answer type is derived from the system and reformulate the question it has semantically equivalent multiple question, Question processing module given a natural language question as input, the question processing module it is also required to, i) Analyze is the processing of in order to represent the main information that is ask by the user question. Analysis of the question is to process of question looking at its main task ii) Classify it is the order of the user asking the question type. Question classifies is based on a question mapping iii) Reformulateit is the transformation of question into queries for the information retrieval (IR) to finally pass a set of query terms to the document processing system. It is also used to perform the information retrieval (IR) (AlChalabi, et al.,2015).

\section{3) Document Processing}

It is referred to as paragraph indexing module, the goal of document processing module is create set of candidate ordered paragraphs that contains the answer and accessing to this goal as order type, the document processing module is required for, i) Retrieve it is a user submitted question that is relevant to set off ranked document, ii) Filterit is document filtration process is returned by the retrieval system in order to sequence of the number of user document, and neither be amount of candidate text in each document, iii) Order it is the user paragraph to call a set of ranked paragraph according to a plausibility degree of containing the correct answer (Al-Chalabi, et al., 2015).

\section{4) Answer Processing}

It is a process of identifying user answers to ask system, from the set of documents the relevant answer is respond to mostly like user answer question, the question extracting and validating from the set of ordered document passed it to form the document processing module, answer processing module is also required to, I Parsing identifies the answer candidates inside the analyzerequestedpassages. ii) Using a set of heuristics, extract the 
answer by selecting the word or expression that answers the supplied questions. iii) Validate the response by expressing confidence in the result's correctness (Al-Chalabi, et al., 2004). (2015).

5) Answer

The representation of the candidate response from the selected documents that make up the answer is the final component in the basic architecture of the Question Answering System. To examine the contents of the papers, the system that analyses the query to get a typical answer employs a few ways. These tactics can be implemented using the coordinating process, which requires that the candidate response text's text unit contain a string whose semantic form matches the typical answer (Breja, Manvi, et al., 2019).

\section{General component of Question answering system}

Component of Question Answer Systemconsists of three levels of processing (Al-Chalabi, et al., 2015).

1) Question Analysis

The questions posed to the system are processed in the question analysis module to detect and extract information that may be useful to other modules. This is accomplished through two main tasks: A) classification of the question to determine the type of information that the question expects an answer (a date, a quantity, etc.) and B) selection of those elements that will allow the system to locate the document. 
2) Passage Retrieval

The document or passage selection module performs an initial selection of answer-bearing candidate texts using information acquired from the question analysis module. Documentoriented approaches are preferable over passage-oriented approaches. The most basic method of passage retrieval is to use a fixed-size sliding window and recover the most significant bits using classic Information Retrieval techniques.

3) Question Classification

The division of the question into categories that describe the type of answer desired is an important part of the question. The application of sets of criteria that translate patterns of questions into question categories is one of the simplest approaches for classifying questions found in the literature. Regular expression on the surface form is used to express the patterns. The most common method for determining the answer type is to examine the question's interrogative phrases.

\section{Method}

Created a Microsoft form online (Paper Sheet) for collecting the survey for understanding level of question by student, it has some multiple-choice questions (MCQ), true or false, fill in the blank answer in one word, objective type question. The Survey has 20 questions for a paper sheet include student name, time to completion, and points for mark show on the dashboard.

\section{Collection}

Question paper data was collected from 63 students at the school level of survey, each paper sheet contains 20 questions, which include the multiple-choice question, one-word answer, filling the blanks. The main target of the collection of datasets is filling the blank questions 
selected for analysis of data. Because students not type question's answer appropriately such type of question, it means some student not written correctly. Some spelling mistakes happen while writing the answers. Some students also type wrong answer. Snapshot 1 shows the paper sheet for collected data.

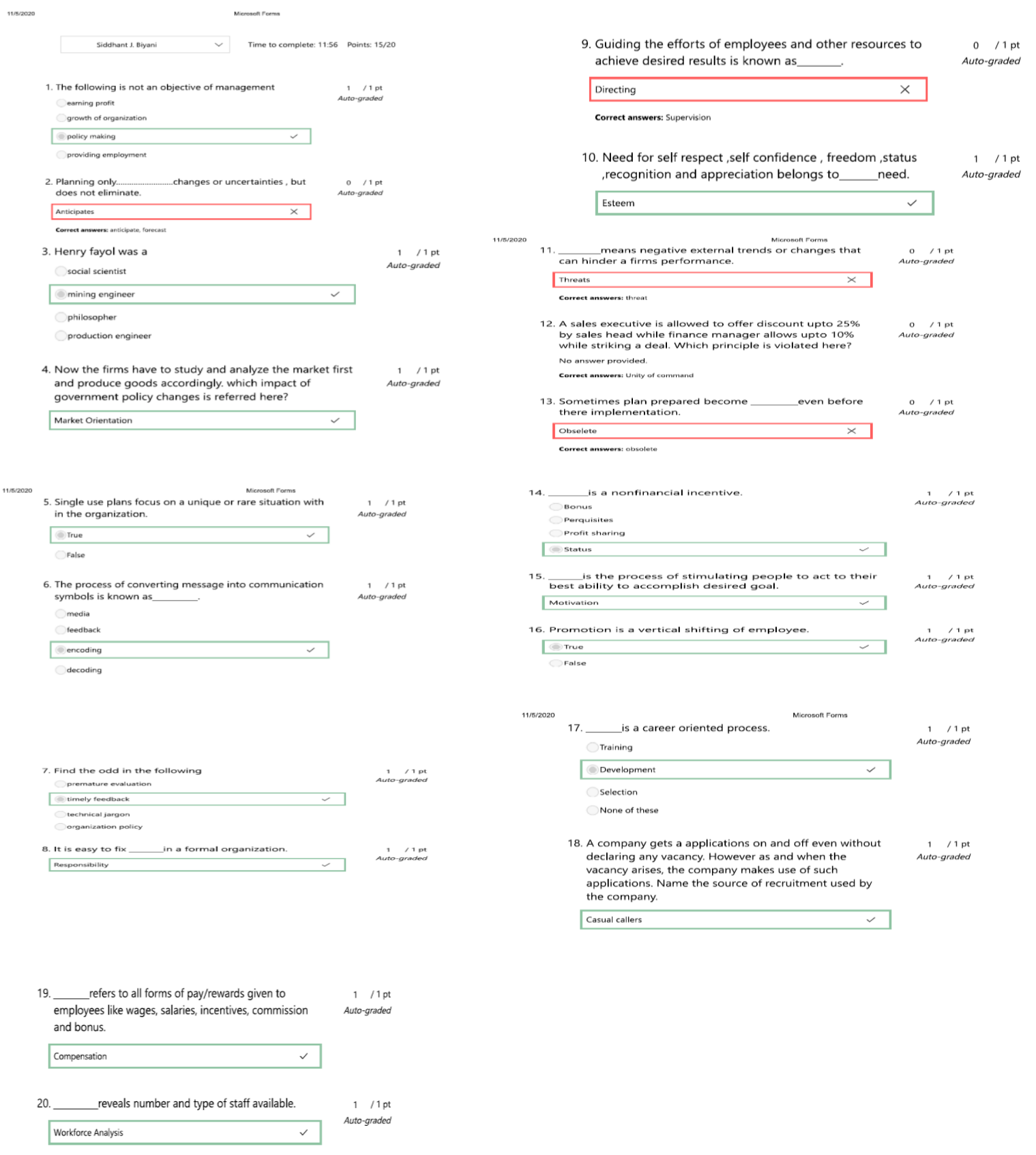

Snapshot 1 Question Paper format for the survey 


\section{Analysis \& Result}

\begin{tabular}{|l|l|l|l|l|l|l|l|}
\hline $\begin{array}{l}\text { Sr. } \\
\text { No. }\end{array}$ & $\begin{array}{l}\text { Question No. \& } \\
\text { Correct Answer }\end{array}$ & $\begin{array}{l}\text { Student } \\
\text { Attempt }\end{array}$ & $\begin{array}{l}\text { Wrong } \\
\text { Answer }\end{array}$ & $\begin{array}{l}\text { Spelling } \\
\text { Mistake } \\
-1 \text { Letter }\end{array}$ & $\begin{array}{l}\text { Spelling } \\
\text { Mistake -2 } \\
\text { Letter }\end{array}$ & $\begin{array}{l}\text { Spelling } \\
\text { Mistake - } \\
3 \text { Letter }\end{array}$ & $\begin{array}{l}\text { Spelling } \\
\text { Mistake - } \\
\text { 4 Letter }\end{array}$ \\
\hline 1 & Anticipate, forecast & 63 & 61 & 1 & 0 & 0 & 0 \\
\hline 2 & Market orientation & 63 & 17 & 2 & 1 & 0 & 0 \\
\hline 3 & Responsibility & 63 & 13 & 3 & 3 & 3 & 1 \\
\hline 4 & Supervision & 63 & 29 & 2 & 4 & 0 & 0 \\
\hline 5 & Threat & 63 & 14 & 37 & 0 & 0 & 0 \\
\hline 6 & Unity Of command & 63 & 27 & 2 & 0 & 0 & 0 \\
\hline 7 & Obsolete & 63 & 36 & 2 & 0 & 0 & 0 \\
\hline 8 & Motivation & 63 & 3 & 1 & 2 & 0 & 0 \\
\hline 9 & Casual callers & 63 & 9 & 10 & 3 & 2 & 0 \\
\hline 10 & Compensation & 63 & 43 & 1 & 0 & 0 & 0 \\
\hline 11 & Workforce analysis & 63 & 33 & 4 & 0 & 0 & 2 \\
\hline
\end{tabular}

Table 1student analysis for correct answer, wrong answer and spell mistake answer.

The given table shows student analysis for correct answer, wrong answer and answer with spell mistake. To understand level of writing for online examination, analysis has been performed by selecting only filling the blank questions. The total number of questions considered was 11 out of 20 questions as shown in above Table 1. The first question contains correct word 'anticipate forecast', attempted by 63 students, 61 students written wrong answer, one student given difference of one letter spell mistake in that question, the second question contain correct word 'market orientation', attempted by 63 students, 17 students written wrong answer, 2 students given difference of one letter and 1 student given difference of two letter spell mistake in that question, third question contain correct word is 
'Responsibility' attempted by 63 students, 13 students written wrong answer, 3 students respectively given difference of one, two and one student gives difference four letter spell mistake in that question, the 'supervision' question contain correct word, attempted by 63 students, 29 students written wrong answer, 2 students given difference of one letter, and 4

students gives difference two letter spell mistake in that question, 'Threat' question contain correct word, attempted by 63 students, 14 students written wrong answer, 37 students given difference of one letter spell mistake in that question, 'Unity of command' question contain correct word, attempted by 63 students, 27 students written wrong answer, 2 students given difference of one word letter spell mistake in that question, 'obsolete' question contain correct word, attempted 63 students, 36 students written wrong answer, 2 students given difference of one word letter spell mistake in that question, 'Motivation' question contain correct word, attempted by 63 students, 3 students written wrong answer, 1 students given difference of one word letter and 2 students gives difference of one word letter spell mistake in that question, 'Casual callers' question contain correct word, attempted by 63 students, 9 students written wrong answer, 10 students gives difference of one word letter, 3 students gives difference of two word letter, and 2 students gives difference of three word letter spell mistake in that question, 'Compensation' question contain correct word attempted by 63 students, 43 students written wrong answer, 1 students gives difference of one word letter spell mistake in that question, 'Workforce analysis' question contain correct word attempted by 63 students, 33 students written wrong answer, 4 students gives difference of one word letter and 2 students gives difference of four word letter spell mistake in that question. 


\section{Conclusion}

Question answering system is generally developed by restricted domain easy to analysis of what type of question user ask to system, it has limited capabilities to developing the question answering system in that domain. Basic architecture of question answering system contains it consist of given processing i.e. candidate question, question processing, document processing answer processing and final candidate answer. The general components of question answering system contains question analysis, passage retrieval, question classification. Question paper data was collected from 63 students at the school level of survey, each paper sheet contains 20 questions, includes multiple-choice question, one-word answer. Out of 20 questions only 11 questions having type fill in the blank considered for analysis. The analysis contains correct word in answer, count of students who attempted question, count of students written wrong answer, count of students with how many letters difference in spell mistake for that question. The main objective of this research is to understand level of writing for online examination, difficulties facing by students while writing answers.

\section{Acknowledgment}

The authors would like to express their gratitude to the Chhatrapati Shahu Maharaj Research Training \& Human Development Institute (SARTHI), Pune, for awarding them a fellowship under the CSRI DST Major Project No.SR/CSRI/71/2015(G), as well as the Computational and Psycholinguistic Research Lab Facility and the Department of Computer Science \& Information Technology, Dr. Babasaheb Ambedkar. 


\section{References}

Dhokrat, Asmita, Hanumant Git, and C. Namrata Mahender (2012)."Assessment of Answers: Online Subjective Examination." Proceedings of the Workshop on Question Answering for Complex Domains.

Gupta, Poonam, and Vishal Gupta (2012)."A survey of text question answering techniques."International Journal of Computer Applications 53, no. 4.

Vijoy, Maria \& Jamal, Sangeetha(2016). SURVEY ON QUESTION ANSWERING SYSTEM.. International Journal of Advanced Research. 4. 1052-1056. 10.21474/IJAR01/1303.

Wikipedia“WhatIs question” (https://en.wikipedia.org/wiki/question).

Allam, Ali Mohamed Nabil, and Mohamed Hassan Haggag (2012)."The question answering systems: A survey." International Journal of Research and Reviews in Information Sciences (IJRRIS) 2, no. 3.

Al-Chalabi, Hani, Santosh Ray, and Khaled Shaalan (2015). "Semantic based query expansion for Arabic question answering systems." In 2015 First International Conference on Arabic Computational Linguistics (ACLing), pp. 127-132. IEEE.

Breja, Manvi, and Sanjay Kumar Jain (2019)."A Survey on Why-Type Question Answering Systems."arXiv preprint arXiv:1911.04879. 